\title{
Depressão, Ansiedade e Atividade de Doença na Artrite Reumatóide
}

\section{Depression, Anxiety and Disease Activity in Rheumatoid Arthritis}

\author{
Antonio Filpi Coimbra da Costa ${ }^{(1)}$, Marco Antonio Alves Brasil ${ }^{(2)}$, Jose Angelo Papi ${ }^{(3)}$, \\ Mario Newton Leitão de Azevedo(4)
}

\section{RESUMO}

Objetivo: Avaliar a prevalência de transtornos psiquiátricos em pacientes com artrite reumatóide (AR), do sexo feminino, e relacionar esses quadros com a atividade de doença. Métodos: Estudo seccional em pacientes do sexo feminino, acima de 18 anos de idade, com diagnóstico de $\mathrm{AR}$, conforme os critérios do American College of Rheumatology (ACR), com graus de incapacidade II a III, com mais de seis meses de doença. Para avaliação da atividade clínica, usou-se o DAS-28 (Disease Activity Score-28), definindo-se como remissão até 2,6 pontos; atividade moderada de 2,6 a 5,1 pontos e atividade intensa acima de 5,1 pontos. A avaliação psiquiátrica foi feita com o Structural Clinical Interiview for the DSM-IV(SCID), entrevista semi-estruturada, com base no DSM-IVTR (Diagnostic and Statistical Manual of Mental Disorders), para diagnosticar a presença de transtornos ansiosos e depressivos. Na análise estatística, incluíram-se o teste do qui-quadrado, teste t, teste de KruskallWallis e o teste de Kendall, com significância de 95\%. Resultados: Em 107 pacientes analisados, $36(33,7 \%)$ apresentaram transtornos psiquiátricos. Em relação à atividade da doença, os dados do DAS28 mostraram: $6(5,6 \%)$ pacientes em remissão; $59(55,1 \%)$ em atividade moderada e $42(39,2 \%)$ com atividade acentuada. A média de pontos do DAS-28 foi de 4,56 entre pacientes sem diagnóstico psiquiátrico e 5,43 entre aqueles com diagnóstico psiquiátrico $(\mathrm{p}=0,001)$. Conclusões: A prevalência de transtornos ansiosos e depressivos entre 107 pacientes com AR foi de 33,7\%. A atividade de doença medida pelo DAS mostrou que pacientes com quadros psiquiátricos apresentavam, em média, maior atividade de doença. Não foram encontradas pessoas com diagnóstico psiquiátrico no grupo de pacientes em remissão.

Palavras-chave: artrite reumatóide, DAS-28, ansiedade, depressão.

\begin{abstract}
Objective: to evaluate the prevalence of psychiatric disorders in female patients, diagnosed with Rheumatoid Arthritis and relate this disorder to disease activity. Methods: sectional trial study with female patients, aged 18 years old or older, diagnosed with Rheumatoid Arthritis (following American College of Rheumatology - ACR criteria), more than six months of disease and incapacity levels II and III. Disease activity was evaluated with DAS 28 (Disease Activity Score-28) (remission up to 2.6/ moderate activity 2.6 to 5.1 /severe degree over 5.1). Psychiatric evaluation was conducted using SCID protocol; interviews were held to diagnose the presence of depression and anxiety disorders following the Diagnostic and Statistical Manual of Mental Disorders (DSM-IV TR). Statistical analysis included the Kruskal-Wallis test and the Kendall test with a significance level of 95\%. Results: In the 107 patients analyzed, 36 subjects (33.7\%) presented psychiatric disorders and 71(66.4\%) no psychiatric disorders). Disease activity - DAS 28 score results; remission: 6 patients (5.6\%); moderate activity: 59 patients (55.1\%); severe disease: 42 patients (39.2\%). The relationship between psychiatric disorders and disease activity (DAS 28) demonstrated: patients without psychiatric disorders - DAS 28 score: 4.56 and patients with psychiatric disorders, DAS 28 score: 5.43 ( $p=0.001)$. Conclusions: The prevalence of depression and anxiety disorders among 107 patients with Rheumatoid Arthritis was 33.7\%. Disease activity, evaluated by the DAS 28 score, was higher in the group of patients with psychiatric disorders. No patients with psychiatric disorders were identified in the group in clinical remission.
\end{abstract}

Keywords: rheumatoid arthritis, DAS 28, anxiety, depression.

\footnotetext{
Recebido em 13/8/2007. Aprovado, após revisão, em 04/1/2007. Declaramos a inexistência de conflitos de interesse. Universidade Federal do Rio de Janeiro, Hospital Universitário Clementino Fraga Filho

1. Mestre em Clínica Médica, médico do Serviço de Saúde Mental do Hospital Universitário Clementino Fraga Filho da Universidade Federal do Rio de Janeiro (UFRJ).

2. Professor adjunto do Departamento de Psiquiatria e Medicina Legal da Faculdade de Medicina da UFRJ e chefe do Serviço de Psiquiatria e Psicologia Médica do HUCFF-UFRJ.

3. Professor titular do Departamento de Clínica Médica da Faculdade de Medicina da UFRJ.

4. Professor doutor do Departamento de Clínica Médica da Faculdade de Medicina da UFRJ e chefe do Serviço de Reumatologia do HUCFF-UFRJ

Endereço para correspondência: Antonio Filpi Coimbra da Costa, Rua Conde de Bonfim 344, bloco 2, 801, CEP 20520-054, Rio de Janeiro, RJ, e-mail: anfilpi@terra.com.br
} 


\section{INTRODUÇÃO}

A artrite reumatóide (AR) é uma doença inflamatória articular de evolução crônica, caracterizada por episódios dolorosos e deformidades físicas, com conseqüentes limitações no trabalho e nas atividades cotidianas.

A AR apresenta uma prevalência de transtornos depressivos e ansiosos acima da média habitualmente encontrada na população em geral, variando de $13 \%$ a $47 \%^{(1,2)}$. Essa grande diferença provavelmente se deve à variedade das populações estudadas e também ao uso de questionários diferentes para determinação da presença de sintomas depressivos ou ansiosos.

Não há consenso na literatura sobre a origem da maior prevalência dos sintomas psiquiátricos na AR. Estes poderiam ocorrer em razão de seqüelas de uma doença incapacitante ou da própria atividade clínica de uma doença inflamatória crônica. Porém é importante ressaltar que as alterações do estado de humor agravam as queixas dos pacientes, dificultando a continuidade do atendimento e, muitas vezes, piorando o quadro evolutivo ${ }^{(3)}$. Essa sobreposição pode causar distorções em sua avaliação e eventualmente influenciar na condução do tratamento.

O objetivo deste estudo foi verificar a prevalência de transtornos psiquiátricos e sua possível relação com a atividade de doença, medida por intermédio do Disease Activity Score - DAS-28 $8^{(4)}$, em pacientes ambulatoriais com AR.

\section{PACIENTES E MÉTODOS}

Participaram do estudo pacientes com AR, segundo os critérios da American College of Rheumatology (ACR) ${ }^{(5)}$, em tratamento no ambulatório de Reumatologia do Hospital Universitário Clementino Fraga Filho da Universidade Federal do Rio de Janeiro (HUCFF-UFRJ).

Foram convidadas as pacientes que aguardavam atendimento na sala de espera e que preenchessem os critérios de inclusão e exclusão. Todas as participantes assinaram o Termo de Consentimento Livre e Esclarecido.

Era realizada, em primeiro lugar, entrevista com psiquiatra, seguida pela consulta regular com reumatologista, quando eram feitos o exame clínico e reumatológico e aplicava-se o protocolo para avaliação da atividade de doença , por meio do DAS-28 - Disease Activity Score 28.

Os dados dos questionários e o diagnóstico feitos pelo psiquiatra não eram do conhecimento do reumatologista. O psiquiatra, por sua vez, só tomava conhecimento do resultado do DAS após sua conclusão.
Foram considerados critérios para inclusão: sexo feminino com diagnóstico de AR, segundo os critérios do American College of Rheumatology, idade mínima de 18 anos, grau de incapacidade funcional II ou III ${ }^{(6)}$, em tratamento para AR (drogas modificadoras, antiinflamatórios não-hormonais e corticosteróides) e sem quadros psicóticos ou demências.

\section{CRITÉRIOS DE EXCLUSÃO}

Sexo masculino, idade inferior a 18 anos, tempo de doença com duração inferior a seis meses, classe funcional I e IV, doença psiquiátrica preexistentes, doenças inflamatórias do tecido conjuntivo diferentes da AR, artrite infecciosa, endocrinopatia, neuropatia primária, seqüelas de doença neurológica, deformidades físicas secundárias a doenças diferentes da AR.

\section{EXAMES LABORATORIAIS}

Solicitou-se para avaliação de atividade da doença a velocidade da sedimentação das hemácias pelo método Westergreen, velocidade de hemossedimentação (VHS), a fim de realizar o cálculo do DAS-28. O VHS é feito regularmente 15 dias antes da consulta marcada.

\section{AVALIAÇÃO CLÍNICA}

Para avaliar o estado clínico das pacientes, foi usado o Disease Activity Score 28 (DAS-28) ${ }^{(7)}$, índice composto por quatro variáveis: velocidade de hemossedimentação (VHS), número de articulações dolorosas e inflamadas entre 28 articulações e o grau de comprometimento causado pela doença, por meio de uma escala analógica visual, preenchida pelo doente.

Parâmetros para avaliação da atividade da doença pelo DAS- $28^{(8)}$ : até 2,6 - casos em remissão; de 2,6 a 5,1 atividade moderada de doença; acima de 5,1 - atividade intensa de doença.

\section{AVALIAÇÃO PSIQUIÁTRICA}

Todas as pacientes foram entrevistadas pelo mesmo psiquiatra. A avaliação psiquiátrica foi realizada aplicandose o Structural Clinic Interview for the DSM-IV - SCID - versão clínica ${ }^{(9)}$, que consiste em uma entrevista psiquiátrica semi-estruturada, com a finalidade de estabelecer diagnósticos conforme o Manual Diagnóstico e Estatístico de Transtornos Mentais da Associação Psiquiátrica Americana Diagnostic and Statistical Manual of Mental Disorders

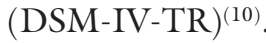


Para fins de pesquisa, foram consideradas como tendo transtornos psiquiátricos todas as pacientes que, no momento da entrevista, preenchiam critérios para inclusão em qualquer um dos itens do DSM-IV-TR.

\section{ANÁLISE ESTATÍSTICA}

Os testes usados foram: mediana, média, desvio-padrão, teste de Kruskall-Wallis e teste de Kendall. Nível de significância de 95\%; valores de p iguais ou inferiores a 0,05 foram considerados estatisticamente significativos.

O teste de Kendall foi usado para verificar se a distribuição de pacientes com e sem diagnóstico psiquiátrico entre os três níveis de gravidade do DAS-28 era estatisticamente significativa ou não. Não foi utilizado o teste do qui-quadrado porque havia mais de $25 \%$ de células com número esperado abaixo de 5 .

$\mathrm{Na}$ avaliação das médias dos componentes do DAS28 , entre pacientes com e sem transtornos depressivos ou ansiosos, viu-se que esses dados não tinham distribuição normal, o que levou à escolha de um teste não paramétrico (Kruskall-Wallis). Foi utilizado o pacote estatístico do programa SPSS.

\section{RESULTADOS}

As pacientes durante a entrevista relataram que só apresentaram transtornos psiquiátricos após o surgimento da AR.

Em virtude do pequeno número amostral não se fez distinção entre pacientes com transtornos depressivos ou ansiosos. Os dados demográficos e clínicos das pacientes encontram-se na tabela 1 . Observou-se uma associação estatisticamente significativa $(\mathrm{p}<0,001)$ entre a média do DAS-28 e a presença de diagnóstico psiquiátrico, sendo a gravidade do quadro clínico de AR maior entre os pacientes com ansiedade e depressão (Tabela 2).

A associação entre os elementos que compõem o DAS-28, tomados isoladamente, e a presença ou ausência de transtornos psiquiátricos só não foi significativa para o VHS (Tabela 3).
TABELA 1

DAdOS DEMOGRÁFICOS E CLÍNICOS DA AMOSTRA DAS 107 PACIENTES COM ARTRITE REUMATÓIDE

\begin{tabular}{lcc}
\hline & Média & Mediana \\
\hline Idade (em anos) & $54,89 \pm 12,51$ & 56,5 \\
Tempo de doença (em anos) & $12,07 \pm 8,37$ & 10 \\
DAS-28 & $4,8562 \pm 1,2926$ & 4,8 \\
\hline
\end{tabular}

TABELA 2

DisTRIBUIČ̃̃O DOS DIAGNÓSTICOS DE TRANSTORNOS PSIQUIÁTRICOS NA AMOSTRA DE PACIENTES DO SEXO FEMININO COM ARTRITE REUMATÓIDE APÓS A APLICAÇÃO DO QUESTIONÁRIO DSM-IV SCID ( $\mathrm{N}=107)$

\begin{tabular}{lcc}
\hline Diagnóstico psiquiátrico & Número de pacientes & DAS-28 \\
\hline Com diagnóstico psiquiátrico & $36(33,7 \%)$ & $5,43(1,04)$ \\
Sem diagnóstico psiquiátrico & $71(66,3 \%)$ & $4,53(1,31)$ \\
Total & $107(100 \%)$ & \\
\hline
\end{tabular}

$\mathrm{p}=0,001$, teste $\mathrm{t}$

Significância estatística $p<0,05$

A prevalência de atividade moderada da AR avaliada pela DAS-28 foi a mais alta. Entre os pacientes com DAS mais alto, encontrava-se a maior prevalência de pacientes com diagnósticos de ansiedade e depressão. O teste de Kendall mede a consistência da distribuição dos dados ao longo de variáveis ordenadas, como no caso do DAS (Tabela 4).

TABELA 4

Comparação do VAlor do DAS-28 de PaCientes COM ARTRITE REUMATÓIDE COM A PRESENÇA OU NÃO DE DIAGNÓSTICO PSIQUIÁTRICO

\begin{tabular}{lccc}
\hline & \multicolumn{3}{c}{ DAS-28 } \\
\cline { 2 - 4 } & $<\mathbf{2 , 6}$ & $\mathbf{2 , 6 - 5 , 1}$ & $>\mathbf{5 , 1}$ \\
\hline Sem diagnóstico psiquiátrico & 6 & 45 & 20 \\
& $(100 \%)$ & $(76,3 \%)$ & $(47,6 \%)$ \\
Com diagnóstico psiquiátrico & 0 & 14 & 22 \\
& & $(23,7 \%)$ & $(52,4 \%)$ \\
\hline
\end{tabular}

Teste de Kendall, $p<0,05$, nível de significância de 95\%

TABELA 3

AsSOCIAÇÃo ENTRE PACIENTES COM E SEM TRANSTORNOS PSIQUiÁTRICOS E AS DIVERSAS VARIÁVEIS QUE COMPÕEM O DAS-28

\begin{tabular}{lcccc}
\hline Diagnóstico psiquiátrico & VHS (média) & Avaliação do paciente (média) & Articulações dolorosas (média) & Articulações inflamadas (média) \\
\hline Sem diagnóstico & 40,93 & 6,320 & 4,28 & 2,69 \\
Com diagnóstico & 45,58 & 8,942 & 6,42 & 3,22 \\
Teste Kruskall-Wallis & 0,275 & 0,002 & 0,031 & 0,024 \\
p valor & & & & \\
\hline
\end{tabular}

Significância estatística $p<0,05$ 


\section{DISCUSSÃO}

A prevalência de transtornos psiquiátricos (depressão e ansiedade) encontrada na amostra, usando-se a entrevista semi-estruturada, foi de $33,7 \%$ dos casos, dentro da média encontrada em outros estudos, embora a maioria das pesquisas utilize questionários de sintomas, como em Escalante et al. ${ }^{(11)}$, Abdel Nasser et al. ${ }^{(12)}$, Velásquez et al..$^{(13)}$ e Pincus et al. ${ }^{(14)}$.

Em alguns trabalhos nos quais se usaram questionários ou entrevistas semi-estruturadas, com diagnóstico psiquiátrico com base no DSM ou no CID, obtiveram-se prevalências mais altas, como nos casos de El-Mieddany et al. ${ }^{(15)}(66,2 \%$ da amostra foi diagnosticada como tendo depressão e $70 \%$, quadros de ansiedade) e Isik et al. ${ }^{(16)}(70,8 \%$ do total dos pacientes com AR apresentavam algum transtorno, ansioso ou depressivo). A exceção foi o trabalho de Suárez-Mendonza et al., no México, que encontraram resultado de $37,14 \%^{(17)}$.

Procurando-se associações entre os componentes do DAS-28 e a presença de quadros depressivos e ansiosos (Tabela 3), verificou-se que o único componente que não mostrou significância estatística foi o VHS. Essa medida foi a mais inespecífica dentre os elementos que compõem o índice (ver Tabela 3).

O DAS-28, ao associar diversos dados, permite uma avaliação global da doença, diferente do que ocorre quando se toma cada medida clínica isoladamente e tenta-se associála ao desempenho psíquico. Um dado importante no uso do DAS-28 é a avaliação feita pelo paciente, que procura obedecer à idéia de tomar a doença como um fenômeno biopsicossocial.

Por outro lado, a avaliação subjetiva do DAS- 28 pode ser confundida com o estado afetivo dos pacientes, provocando um viés de associação positiva entre graus clínicos mais graves

\section{REFERÊNCIAS}

1. Dickens C, Creed F: The burden of depression in patients with rheumatoid arthritis. Rheumatology 40: 1327-30, 2001.

2. Velasquez X, Pizarro C, Pizarro P, Massardo L: La depresión en artritis reumatoídea. Reumatología 18(2): 49-52, 2002.

3. Cavanaugh SVA, Furlanetto LM, Creech SD, Powell LH: Medical illness, past depression, and present depression: a predictive triad for in-hospital mortality. The Am J Psyc 158: 43-8, 2001.

4. Désirée MFM, Heidje VD, Hof MV, Riel PLV, Putte LBV: Validity of single variables and indices to measure disease activity in rheumatoid arthritis. J Rheumatol 20(3): 538-41, 1993.

5. Arnett FC, Edworthy SM, Bloch DA, et al.: The American Rheumatism Association 1987 revised criteria for the classification of rheumatoid arthritis. Arthritis Rheum 31: 315-24, 1988. e a presença dos transtornos psiquiátricos. Artigos que procuraram avaliar o desempenho do DAS-28 comparando com critérios do ACR para remissão da artrite, como o de Fransen $e t$ $a l{ }^{(18)}$, constataram que o índice com e sem avaliação subjetiva apresentava sensibilidade de 90 e 86 , respectivamente, portanto a avaliação subjetiva dos doentes melhorava a capacidade de encontrarem-se casos mais graves. Do mesmo modo, o artigo de Leeb et al.$^{(19)}$ verificou a importância do uso de índices de atividade com avaliação subjetiva para orientar a conduta clínica em relação às manifestações da $\mathrm{AR}$.

Quanto à associação entre atividade de doença medida especificamente pelo DAS- 28 e a presença de quadros ansiosos e depressivos, Hyphantis et al. ${ }^{(20)}$, examinando pacientes com diagnóstico de $\mathrm{AR}$ recente, não encontraram associações entre atividade de doença e sintomas psiquiátricos.

Neste trabalho, observou-se que os piores índices de atividade clínica no DAS-28 estavam associados à presença de alterações psiquiátricas. Nenhum dos pacientes que apresentava quadro de AR em remissão sofria de transtornos depressivos ou ansiosos. Sabe-se que estudos transversais não podem determinar precedências, mas pôde ser observado que mais da metade dos pacientes agrupados no item "atividade intensa de doença" no DAS-28 apresentava transtornos psiquiátricos.

\section{CONCLUSÃO}

A prevalência de transtornos depressivos e ansiosos em pacientes ambulatoriais com artrite reumatóide foi de $33,7 \%$. Houve associação estatisticamente significativa entre pacientes com pior quadro clínico, medido pelo DAS, e o diagnóstico de transtornos depressivos e ansiosos, diagnosticados pelo SCID. Mais estudos, com avaliações longitudinais, são necessários para corroborar ou não essa hipótese.

6. Steinbrocker O, Traeger CH, Batterman RC, et al.: Therapeutic criteria in rheumatoid arthritis. JAMA 140: 659-62, 1949.

7. Désirée MFM, Heidje VD, Hof MV, Riel PLV, Putte LBV: Validity of single variables and indices to measure disease activity in rheumatoid arthritis. J Rheumatol 20(3): 538-41, 1993.

8. Department of Rheumatology of the University Medical Center Nijmegen, disponível em: www.das-score.nl/www.das-score.nl/ index.html. Acessado em: 13/12/2006.

9. SCID (tradução) Tavares, M: A Entrevista Clínica Estruturada para o DSM-IV 1997 - Instituto de Psicologia, Universidade de Brasília.

10. DSM-IV-TR: Manual Diagnóstico e Estatístico de Transtornos Mentais. (tradução) Claudia Dornelles. 4.ed. Texto revisado. São Paulo, Artmed, 2002. 
11. Escalante A, Del Rincón I, Murlow CD: Symptoms of depression and psychological distress among hispanics with rheumatoid arthritis. Arthritis Care and Res 13(3): 156-67, 2000.

12. Abdel-Nasser AM, Abd El-Azim S, Taal E, El-Badawy SA, Rasker JJ, Valkenburg HA: Depression and depressive symptoms in rheumatoid arthritis patients: an analysis of their ocurrence and determinants. Br J Rheumatol 37: 391-7, 1998.

13. Velásquez X, Pizarro C, Pizarro P, Massardo L: La depresión en artritis reumatoídea. Reumatología 18(2): 49-52, 2002.

14. Pincus T, Griffith J, Pearce S, Insberg D: Prevalence of selfreported depression in patients with rheumatoid arthritis. Br J Rheumatol 35: 879-83, 1996.

15. EL-Mieddany, Yasser M, El Rasheed AH: Is Anxiety a more common disorder than depression in Rheumatoid Arthritis? Joint Bone Spine 69: 300-6, 2002.

16. Isik A, Koca SS,Ozturk A, Mermi O: Anxiety and Depression in patients with rheumatoid arthritis. Clin Rheumatol 126 (6): 872-8, 2007.
17. Suárez-Mendonza AA, Cardiel MH, Caballero-Uribe CV, Ortega-Soto HA, Marquez-Marin M: Measurement of depression in mexican patients with rheumatoid arthritis: validity of the beck depression inventory. Arthritis Care and Res 10(3): 194-9, 1997.

18. Fransen J, Creemers MCW, Van Riel PLCM: Remission in rheumatoid arthritis: agreement of the disease activity score (DAS28) with the ARA preliminary remission criteria. Rheumatology 43: 1252-5, 2004.

19. Leeb BF, Andel I, Leder S, Leeb BA, Rintelen B: The patient's perspective rheumatoid arthritis disease activity indexes. Rheumatology 44: 360-5, 2005.

20. Hyphantis TN, Bai M, Siafaka V, Geogiardis AN, Voulgari PV, Mavreas V, Drosos AA: Psychological distress and personality traits in early rheumatoid arthritis: a preliminary survey. Rheumatol Int 26: 828-36, 2006. 